\title{
Primary HHV-8 (-) Effusion-Based Non-Germinal Center B Cell Diffuse Large B Cell Lymphoma Successfully Treated with Standard Anthracycline-Based Chemoimmunotherapy
}

\author{
Justin Kuhlman (DD' \\ Muhamad Alhaj Moustafa (iD) ${ }^{2}$ \\ Liuyan Jiang (D) ${ }^{3}$ \\ Han W Tun (D) ${ }^{2}$ \\ 'Department of Internal Medicine, Mayo \\ Clinic, Jacksonville, FL, USA; ${ }^{2}$ Division of \\ Hematology and Medical Oncology, Mayo \\ Clinic, Jacksonville, FL, USA; \\ ${ }^{3}$ Department of Pathology and \\ Laboratory Medicine, Mayo Clinic, \\ Jacksonville, FL, USA
}

\begin{abstract}
Effusion-based lymphomas (EBL) are usually high-grade B cell non-Hodgkin's lymphomas which involve effusion fluid in a body cavity, typically presenting as a pleural effusion, without evidence of disease elsewhere. They are most frequently seen in HIVinfected individuals and are biologically driven by human herpesvirus- 8 virus (HHV-8). HHV-8 (+) EBL is recognized as primary effusion lymphoma (PEL) under the World Health Organization classification. HHV-8 (-) EBL has been reported in association with Hepatitis $\mathrm{C}$ virus (HCV) infection, Epstein-Barr virus (EBV) infection, fluid overload, liver cirrhosis, renal dysfunction, cardiac arrhythmias, myocardial infarction, and heart failure. These cases can be labeled as primary EBL (PEBL). We describe a non-germinal center B cell diffuse large B cell lymphoma (NGCB-DLBCL) presenting as PEBL in an immunocompetent 81year-old male who had an extensive cardiac history and tested negative for HIV, HHV-8, and EBV. He was treated with thoracentesis and standard anthracycline-based chemoimmunotherapy and has remained in complete remission for over $51 / 2$ years since his original diagnosis. Our case indicates that NGCB-DLBCL can present as PEBL and is potentially curable with the standard chemoimmunotherapeutic approach.
\end{abstract}

Keywords: HHV-8 negative EBL, non-germinal center diffuse large B cell lymphoma, primary effusion-based lymphoma, primary effusion lymphoma

\section{Introduction}

Diffuse large B-cell lymphoma (DLBCL) can frequently involve extra nodal tissues in a secondary process, including body cavities. ${ }^{1}$ On rare occasions, however, such lymphomatous involvement of a body cavity can develop as a primary malignancy without evidence of lymphoma elsewhere. The 2016 revised WHO classification of lymphoid neoplasms describes primary effusion lymphoma (PEL) as a large B-cell lymphoma universally associated with human herpesvirus- 8 (HHV-8) infection. ${ }^{2}$ Conversely, primary HHV-8 negative effusion-based lymphoma (HHV-8 (-) EBL) is a distinct category of non-Hodgkin lymphoma (NHL) characterized by the absence of HHV-8 infection and has only been reported in various case reports, case series, and literature reviews. ${ }^{3-23}$ HHV-8 (-) EBL typically manifests as a primary serous effusion in a body cavity without a distinct tumor mass and is often discovered in immunocompetent hosts who are HIV negative. ${ }^{24}$ HHV-8 (-) EBL has been reported in
Correspondence: Han W Tun Mayo Clinic Florida, Division of Hematology and Oncology, 4500 San Pablo Road S, Jacksonville, FL, 32224, USA

Tel + 1904-953-2000

Email tun.han@mayo.edu 
association with hepatitis $\mathrm{C}$ virus (HCV) infection, EpsteinBarr virus (EBV) infection, fluid overload, liver cirrhosis, renal dysfunction, cardiac arrhythmias, myocardial infarction, and heart failure. ${ }^{5,7-9,16,25}$ Herein, we describe a rare case of NGCB-DLBCL presenting as primary HHV-8 (-) EBL in an immunocompetent 81-year-old male with a complex cardiac history who has been effectively cured following treatment with thoracentesis and anthracyclinebased chemoimmunotherapy.

\section{Case Presentation}

An 81-year-old male with coronary artery disease, valvular heart disease status post mitral valve replacement and tricuspid annuloplasty ring implant, coronary artery bypass graft, persistent atrial fibrillation, hypertension, and congestive heart failure with preserved ejection fraction (EF 60\%) presented to his cardiologist's office complaining of increased fatigue, cough, orthopnea, and exertional dyspnea for 3-4 weeks. He denied any significant weight loss, drenching sweats, increased lymph node size, fevers, or chills. Eight months prior to current presentation during hospitalization for a heart failure exacerbation, the patient was noted to have small bilateral pleural effusions (left greater than right) and underwent left-sided thoracentesis at that time, which revealed no evidence of infection or malignancy. Only one month following that time, the patient underwent mitral valve replacement surgery and during the procedure, the patient underwent bilateral drainage for reaccumulating pleural effusions. Only one month prior to current presentation, the patient was again noted to have another right-sided pleural effusion accredited to heart failure, and the decision was made to up-titrate the patient's diuretic regimen instead of undergoing thoracentesis.

Upon current presentation, chest $\mathrm{x}$-ray reconfirmed a large right-sided pleural effusion, and the patient subsequently underwent repeat diagnostic and therapeutic thoracentesis with serous fluid removed. Cytology was positive for large lymphoma cells (Figure 1A and B). By immunostaining, lymphoma cells were positive for CD20, PAX-5, BCL-2, BCL-6, and MUM-1, and were negative for CD138, CD10, and MYC. The Ki-67 proliferation index was high at $>90 \%$ (Figure 1C-H). Fluorescence in situ hybridization (FISH) testing on lymphoma cells revealed that $55 \%$ of nuclei had three intact copies of MYC located at 8q24, indicative of trisomy 8 or an $8 \mathrm{q}$ duplication. Pathologic findings were consistent with NGCB-DLBCL. An extensive infectious workup, including Epstein-Barr virus (EBV) in situ hybridization testing, HHV-8 immunostaining, hepatitis $\mathrm{C}$ virus RNA quantitative serum testing, hepatitis B core antibody testing, HIV antibody screening, and QuantiFERON Tb-gold analysis were all negative. Computed tomography (CT) scan of the chest, abdomen, and pelvis showed no evidence of pleural-based abnormalities or lymphadenopathy. Full-body positron emission tomography (PET) scan further demonstrated no evidence for any fluorodeoxyglucose (FDG) avid process (Figure 2). Bone marrow aspirate and biopsy was negative for lymphoma. The diagnosis of primary effusionbased lymphoma (PEBL) was made.

Due to the patient's age and with concern towards his ability to tolerate standard anthracycline-based

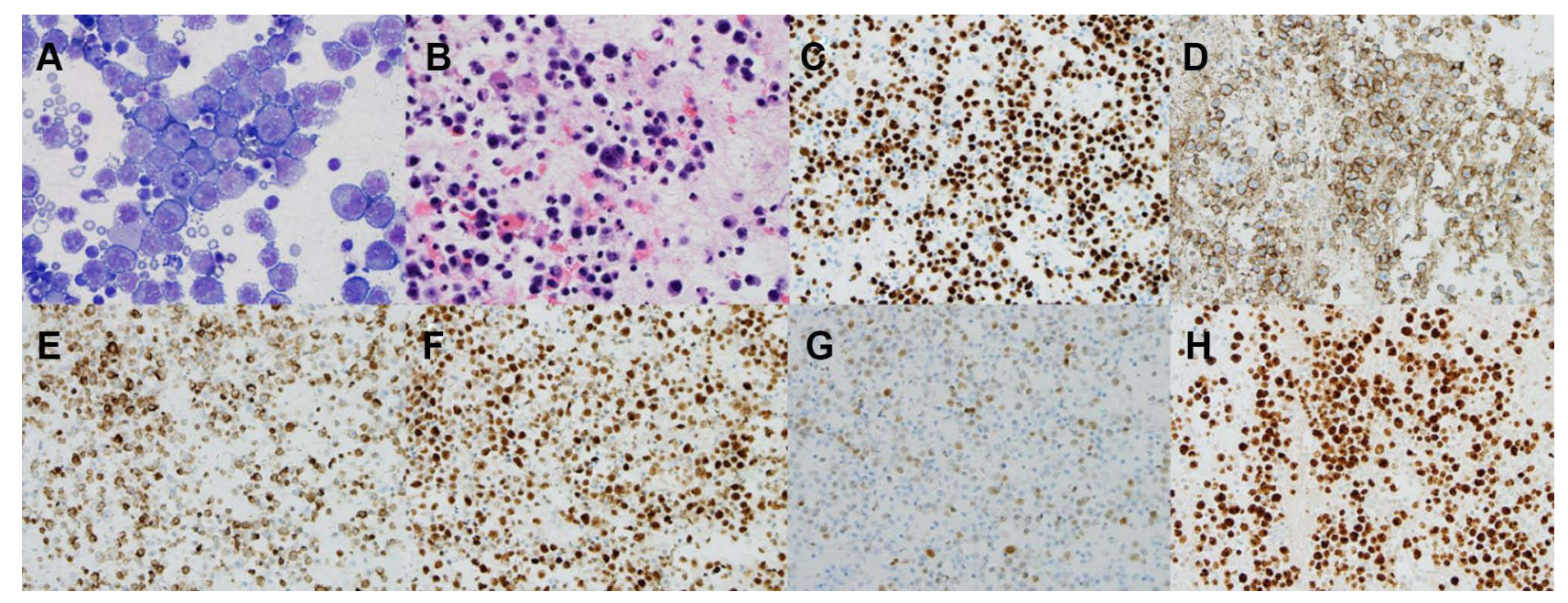

Figure I The pleural fluid contained numerous large atypical lymphocytes on the cytospin slide ((A) Giemsa and Wright stain, $x$ 50) and cell block ((B) Hematoxylin and eosin stain, $x$ 40). Immunohistochemistry studies showed the lymphocytes were positive for PAX 5, CD20, BCL2, BCL6, MUMI (weak) with a high proliferative rate by Ki$6790 \%((\mathbf{C}-\mathbf{H}) \times 20)$. 


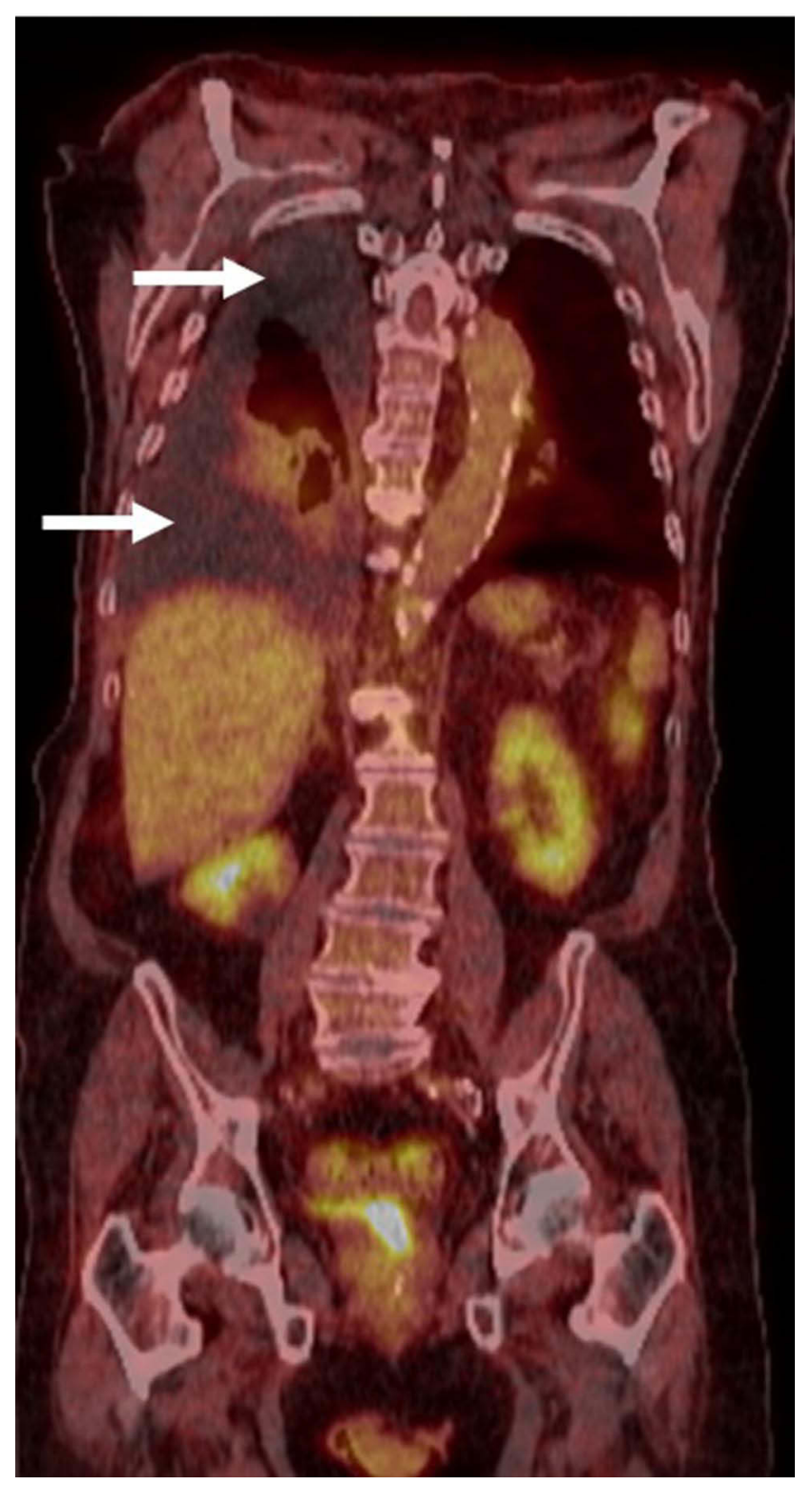

Figure 2 PET scan demonstrated no abnormal FDG processes throughout the body. Large right-sided pleural effusion was present, but without any hypermetabolic activity (arrows).

chemoimmunotherapy with R-CHOP (rituximab, cyclophosphamide, doxorubicin, vincristine, and prednisone), the patient was initially treated with one dose of rituximab $(375 \mathrm{mg} / \mathrm{m} 2)$ as single agent therapy followed by rituximab and cyclophosphamide $(750 \mathrm{mg} / \mathrm{m} 2)$ for two cycles, which were well tolerated. He was then transitioned to $\mathrm{R}-\mathrm{CHOP}$ with a $30 \%$ reduction in doxorubicin dosing due to concerns for cardiac toxicity. Before the second cycle of R-CHOP, he was noted to have a persistent moderate right-sided pleural effusion, and he underwent thoracentesis with negative cytology for lymphoma cells. The patient subsequently completed an additional 5 cycles of
R-CHOP (for a total of 6 cycles). CT chest, abdomen, and pelvis following treatment completion revealed no suspicious lymphadenopathy with a small stable right-sided pleural effusion. Thereafter, the patient was monitored with surveillance imaging every 4-6 months and did not require any further thoracenteses due to resolution of his previous pleural effusion. Currently, he remains in complete remission $5 \frac{1}{2}$ years after the time of his original diagnosis.

\section{Discussion}

High-grade B cell lymphomas primarily involving effusion fluid can be divided into primary effusion lymphoma (PEL), which is positive for HHV-8 and usually found in association with HIV infection, and primary effusionbased lymphoma (PEBL), which is negative for HHV-8. Although they share similar clinical presentations, there are several key differentiating features between these two entities. PEBL notably occurs in patients above 70 years of age while the median diagnostic age of PEL has been reported to be as low as 44 years. $^{5,7,9,22}$ Unlike PEL, which often carries a poor prognosis with an estimated median survival of 6 months, ${ }^{26}$ PEBL is associated with better survival outcomes. The largest review series involving 64 patients with PEBL recently reported an overall survival (OS) of $84.7 \%$ at two years. ${ }^{7}$ PEBL has also been proven to show better response rates to chemotherapy in comparison to PEL despite similar treatment profiles, with first-line therapy typically consisting of CHOP-like therapy with or without rituximab. Alexanian et al also discovered a significant difference in treatment outcomes in response to systemic chemotherapy among PEBL and PEL patients when they reported a treatment response in $82.1 \%$ and $36.9 \%$ of patients, respectively. ${ }^{5}$ This same review also demonstrated that PEBL carried a much higher rate of CD20 expression compared to PEL as $71 \%$ of PEBL patients demonstrated CD20 positivity versus only $15 \%$ of PEL patients. In the case of PEBL, it has been further reported that increased age and CD20 positivity appear to be favorable independent prognostic factors, both of which were present in our patient. ${ }^{10}$ For this reason, and as we and others have suggested elsewhere, CD20 targeting therapy with rituximab in combination with thoracentesis of effusion fluid seems to be an acceptable alternative treatment option in CD20 positive patients who cannot withstand CHOP-like chemotherapy due to increased age or an inadequate performance functional status. ${ }^{11,14,18}$ Our patient was able to successfully complete R-CHOP after 
initial treatment with rituximab monotherapy and rituximab-cyclophosphamide.

Other reports of PEBL have also interestingly documented complete remission following thoracentesis alone as well as spontaneous regression without any treatment whatsoever, with remissions lasting up to multiple years in certain cases. $^{7,9,19,21,27}$ This response of PEBL to drainage alone is in clear discrepancy with PEL as Alexanian et al's review notably reported that $70 \%$ of PEBL patients experienced partial or complete remission following thoracentesis alone as compared to only $18 \%$ of PEL patients..$^{5}$ Although thoracentesis alone is a promising treatment option, we propose that thoracentesis alongside R-CHOP should be used in eligible patients since the data supporting thoracentesis alone is quite limited at this time. Moreover, most of the available data has demonstrated prolonged remissions primarily following reception of $\mathrm{CHOP}$-like chemotherapy with or without rituximab. Most notably, in Kaji et al's review, $86 \%$ of patients who received systemic CHOP or CHOP-like therapy demonstrated an overall response of $95 \%$, with $73 \%$ achieving complete remission. ${ }^{7}$ These and other reports, including the current case, suggest that CHOP or CHOPlike chemotherapy with or without rituximab should be the first-line treatment for eligible patients with PEBL. It appears that therapeutic agents in R-CHOP can penetrate and achieve therapeutic concentrations in the effusion fluid, thereby killing lymphoma cells as well as modulating effusion fluid milieu. Nonetheless, the interesting phenomenon of spontaneous remission following thoracentesis alone, in combination with PEBL's favorable prognosis when compared to PEL, seems to suggest a unique mechanism of action in the lymphomagenesis of this entity that is clearly distinct from PEL. These findings suggest that PEBL is more biologically dependent on effusion fluid milieu when compared to PEL which is driven by HHV-8. Thoracentesis may be therapeutic in the case of PEBL due to the clearance of lymphoma cells as well as removal of the effusion fluid which seems to promote lymphomagenesis.

Although the exact etiology of PEBL remains undetermined, multiple conditions have been found in association with this distinct entity, including fluid overload states, HCV infection, EBV infection, cardiac arrhythmias, myocardial infarction, heart failure, renal dysfunction, liver cirrhosis, and hypothyroidism. ${ }^{5,7-9,16,25}$ In two of the largest reviews analyzing PEBL patients, approximately half of cases had fluid overload preceding a lymphoma diagnosis. $^{5,7}$ This is also the case in our patient. It is important that clinicians include primary lymphomatous effusions in their differential diagnosis when patients present with recurrent pleural effusions as PEBL can be easily misdiagnosed as fluid overload. It is possible that conditions predisposing to fluid overload lead to effusions with milieu conducive to lymphomagenesis. Localized inflammation in a confined environment of effusion may play a role in the lymphomagenesis of PEBL, analogous to pyothorax-associated lymphoma (PAL) which develops in the setting of chronic uncontrolled pyothorax. Unlike PAL, PEBL typically lacks evidence of inflammation, such as serosal membrane thickening, and the effusion fluid is typically serous, not exudative nor purulent. Moreover, PAL usually occurs in those with a history of pulmonary tuberculosis and is strongly associated with EBV infection and positivity for EBV latent genes (LMP-1, EBNA-1, EBNA-2). Our patient demonstrated no evidence of serosal membrane thickening and his pleural fluid was serous, not purulent. He further lacked any history of tuberculosis and tested negative on QuantiFERON Tb-gold analysis and EBV in situ hybridization. Also included in our patient's differential diagnosis was extranodal Burkitt lymphoma, but the absence of MYC rearrangement on FISH analysis and the presence of large cell morphology on histological analysis was consistent with NGCB-DLBCL. Cytogenetically, trisomy 8 has been reported in certain cases of PEBL. ${ }^{12,16,17,23,28,29}$ Our patient likely possesses trisomy 8 as FISH testing revealed three copies of MYC with negative staining for MYC on immunostaining.

\section{Conclusion}

In conclusion, NGCB-DLBCL can present as PEBL in the setting of cardiac conditions predisposing to fluid overload and is potentially curable with the standard anthracyclinebased chemoimmunotherapeutic approach. Therapeutic agents in the R-CHOP regimen appear to have excellent penetration into effusion fluid with significant therapeutic impact. Effusion fluid removal by thoracentesis likely has therapeutic impact via eradication of lymphoma cells as well as removal of fluid milieu which promotes lymphomagenesis. We suggest that both thoracentesis and anthracycline-based chemoimmunotherapy should be employed when treating eligible patients with PEBL.

\section{Ethics and Consent}

Written informed consent was obtained from the patient for the publication of this manuscript and any accompanying images. Institutional approval was not required for publication. 


\section{Funding}

This research received no specific grant from any funding agency in the public, commercial, or not-for-profit sectors.

\section{Disclosure}

The authors declare that there are no conflicts of interest regarding the publication of this manuscript.

\section{References}

1. Møller MB, Pedersen NT, Christensen BE. Diffuse large B-cell lymphoma: clinical implications of extranodal versus nodal presentation - a population-based study of 1575 cases. Br J Haematol. 2004;124(2):151-159.

2. Swerdlow SH, Campo E, Pileri SA, et al. The 2016 revision of the World Health Organization classification of lymphoid neoplasms. Blood. 2016;127(20):2375-2390. doi:10.1182/blood-2016-01-643569

3. Carbone A, Gloghini A. PEL and HHV8-unrelated effusion lymphomas: classification and diagnosis. Cancer Cytopathol. 2008;114 (4):225-227. doi:10.1002/cncr.23597

4. Adiguzel C, Bozkurt SU, Kaygusuz I, Uzay A, Tecimer T, Bayik M. Human herpes virus 8-unrelated primary effusion lymphoma-like lymphoma: report of a rare case and review of the literature. Apmis. 2009;117(3):222-229. doi:10.1111/j.1600-0463.2008.00005.x

5. Alexanian S, Said J, Lones M, Pullarkat ST. KSHV/HHV8-negative effusion-based lymphoma, a distinct entity associated with fluid overload states. Am J Surg Pathol. 2013;37(2):241. doi:10.1097/ PAS.0b013e318267fabc

6. Chen BJ, Chen DYT, Kuo CC, Chuang SS. EBV-associated but HHV8-unrelated double-hit effusion-based lymphoma. Diagn Cytopathol. 2017;45(3):257-261. doi:10.1002/dc.23638

7. Kaji D, Ota Y, Sato Y, et al. Primary human herpesvirus 8-negative effusion-based lymphoma: a large B-cell lymphoma with favorable prognosis. Blood Adv. 2020;4(18):4442-4450. doi:10.1182/ bloodadvances.2020002293

8. Kim Y, Huh J, Yoon S. Primary effusion lymphoma and human herpesvirus 8-negative effusion-based lymphoma: analysis of 17 cases in Korea. Korean J Pathol. 2013;47(Suppl 1):S100.

9. Kobayashi Y, Kamitsuji Y, Kuroda J, et al. Comparison of human herpes virus 8 related primary effusion lymphoma with human herpes virus 8 unrelated primary effusion lymphoma-like lymphoma on the basis of HIV: report of 2 cases and review of 212 cases in the literature. Acta Haematol. 2007;117(3):132-144. doi:10.1159/ 000097460

10. Kubota T, Sasaki Y, Shiozawa E, Takimoto M, Hishima T, Chong J-M. Age and CD20 expression are significant prognostic factors in human herpes virus-8-negative effusion-based lymphoma. Am J Surg Pathol. 2018;42(12):1607-1616. doi:10.1097/ PAS.0000000000001168

11. Matsumoto Y, Nomura K, Ueda K, et al. Human herpesvirus 8-negative malignant effusion lymphoma: a distinct clinical entity and successful treatment with rituximab. Leuk Lymphoma. 2005;46 (3):415-419. doi:10.1080/10428190400018364

12. Saini N, Hochberg EP, Linden EA, Jha S, Grohs HK, Sohani AR. HHV8-negative primary effusion lymphoma of B-cell lineage: two cases and a comprehensive review of the literature. Case Rep Oncol Med. 2013;2013:292301.

13. Takahashi T, Hangaishi A, Yamamoto G, Ichikawa M, Imai Y, Kurokawa M. HIV-negative, HHV-8-unrelated primary effusion lymphoma-like lymphoma: report of two cases. Am J Hematol. 2010;85(1):85-87.
14. Yamaoka O, Matsui T, Nishiyama K, et al. Indolent primary effusion lymphoma-like lymphoma in the pericardium: a case report and review of the literature. $J$ Cardiol Cases. 2019;19(5):148-152. doi:10.1016/j.jccase.2018.12.013

15. Ashihara E, Shimazaki C, Hirai H, et al. Human herpes virus 8-negative primary effusion lymphoma in a patient with a ventriculoperitoneal shunt tube. Int $J$ Hematol. 2001;74 (3):327-332. doi:10.1007/BF02982069

16. Ichinohasama R, Miura I, Kobayashi N, et al. Herpes virus type 8-negative primary effusion lymphoma associated with PAX-5 gene rearrangement and hepatitis $\mathrm{C}$ virus: a case report and review of the literature. Am J Surg Pathol. 1998;22(12):1528-1537. doi:10.1097/ 00000478-199812000-00010

17. Kashiwagi T, Minagawa K, Kawano H, et al. HIV-negative, HHV-8-unrelated primary effusion lymphoma-like lymphoma with genotypic infidelity and c-MYC expression. Ann Hematol. 2014;93 (9):1609-1610. doi:10.1007/s00277-013-1999-3

18. Kuhlman JJ, Alhaj Moustafa M, Tun AJ, Menke DM, Tun HW, Jiang L. Primary age-related EBV-associated effusion-based lymphoma successfully treated with rituximab and thoracentesis. Clin Case Rep. 2021;9(5):e03971. doi:10.1002/ccr3.3971

19. Nakatsuka S-I, Kimura H, Nagano T, et al. Self-limited effusion large B-cell lymphoma: two cases of effusion lymphoma maintaining remission after drainage alone. Acta Haematol. 2013;130 (3):217-221. doi:10.1159/000350482

20. Rodriguez J, Romaguera JE, Katz RL, Said J, Cabanillas F. Primary effusion lymphoma in an HIV-negative patient with no serologic evidence of kaposi's sarcoma virus. Leuk Lymphoma. 2001;41(12):185-189. doi:10.3109/10428190109057969

21. Terasaki Y, Yamamoto H, Kiyokawa H, et al. Disappearance of malignant cells by effusion drainage alone in two patients with HHV-8-unrelated HIV-negative primary effusion lymphoma-like lymphoma. Int $J$ Hematol. 2011;94(3):279-284. doi:10.1007/ s12185-011-0906-8

22. Usmani A, Walts AE, Patel S, Alkan S, Kitahara S. HHV8-negative effusion based lymphoma: a series of 17 cases at a single institution. $J \quad A m$ Soc Cytopathol. 2015;4(1):37-43. doi:10.1016/j. jasc.2014.10.002

23. Wu W, Youm W, Rezk SA, Zhao X. Human herpesvirus 8-unrelated primary effusion lymphoma-like lymphoma: report of a rare case and review of 54 cases in the literature. Am J Clin Pathol. 2013;140 (2):258-273. doi:10.1309/AJCPHZ3CHO4HUWET

24. Carbone A, Gloghini A. PEL and HHV8-unrelated Effusion Lymphomas: Classification and Diagnosis. Wiley Online Library; 2008.

25. Kim M, Han S, Yu JR, et al. A case of primary effusion lymphoma in a patient with chronic kidney disease. Korean J Med. 2012;83 (3):385-389. doi:10.3904/kjm.2012.83.3.385

26. Swerdlow SH, Campo E, Harris NL, et al. WHO Classification of Tumours of Haematopoietic and Lymphoid Tissues. Vol. 2. Lyon, France: International agency for research on cancer; 2008.

27. Kim M, An J, Yoon SO, et al. Human herpesvirus 8-negative effusion-based lymphoma with indolent clinical behavior in an elderly patient: a case report and literature review. Oncol Lett. 2020;20(6):1.

28. Mendeville M, Roemer MG, van den Hout MF, et al. Aggressive genomic features in clinically indolent primary HHV8-negative effusion-based lymphoma. blood. 2019;133(4):377-380. doi:10.1182/blood-2017-12-822171

29. Ohshima K, Ishiguro M, Yamasaki S, et al. Chromosomal and comparative genomic analyses of HHV-8-negative primary effusion lymphoma in five HIV-negative Japanese patients. Leuk Lymphoma. 2002;43(3):595-601. doi:10.1080/10428190290012100 


\section{Publish your work in this journal}

The Journal of Blood Medicine is an international, peer-reviewed, open access, online journal publishing laboratory, experimental and clinical aspects of all aspect pertaining to blood based medicine including but not limited to: Transfusion Medicine; Blood collection, Donor issues, Transmittable diseases, and Blood banking logistics; Immunohematology; Artificial and alternative blood based therapeutics; Hematology; Biotechnology/nanotechnology of blood related medicine; Legal aspects of blood medicine; Historical perspectives. The manuscript management system is completely online and includes a very quick and fair peer-review system. Visit http://www.dovepress.com/testimonials.php to read real quotes from published authors. 